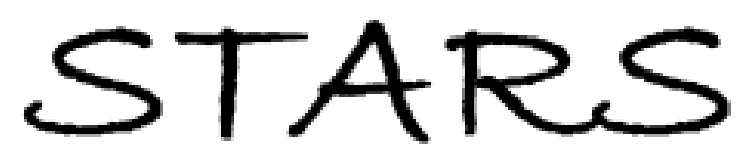

University of Central Florida

STARS

$1-1-2012$

\title{
Wise-Neowise Observations of Active Bodies In The Main Belt
}

James M. Bauer
A. K. Mainzer
Tommy Grav
Russell G. Walker
Joseph R. Masiero

See next page for additional authors

Find similar works at: https://stars.library.ucf.edu/facultybib2010

University of Central Florida Libraries http://library.ucf.edu

This Article is brought to you for free and open access by the Faculty Bibliography at STARS. It has been accepted for inclusion in Faculty Bibliography 2010 s by an authorized administrator of STARS. For more information, please contactSTARS@ucf.edu.

\section{Recommended Citation}

Bauer, James M.; Mainzer, A. K.; Grav, Tommy; Walker, Russell G.; Masiero, Joseph R.; Blauvelt, Erin K.; McMillan, Robert S.; Fernández, Yan R.; Meech, Karen J.; Lisse, Carey M.; Cutri, Roc M.; Dailey, John W.; Tholen, David J.; Riesen, Timm; Urban, Laurie; Khayat, Alain; Pearman, George; Scotti, James V.; Kramer, Emily; Cherry, De'Andre; Gautier, Thomas; Gomillion, Stephanie; Watkins, Jessica; and Wright, Edward L., "Wise-Neowise Observations of Active Bodies In The Main Belt" (2012). Faculty Bibliography 2010s. 2271. https://stars.library.ucf.edu/facultybib2010/2271

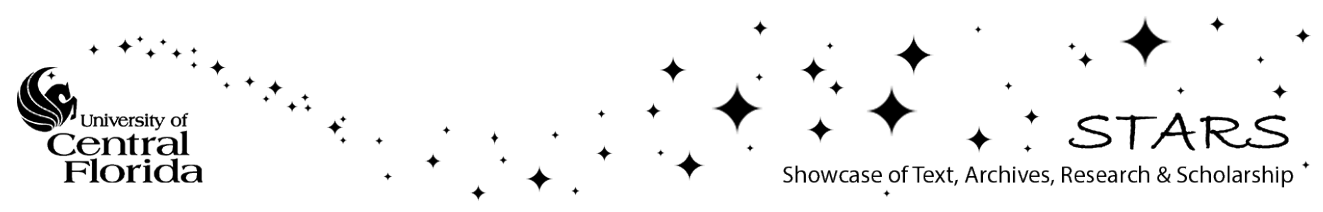




\section{Authors}

James M. Bauer, A. K. Mainzer, Tommy Grav, Russell G. Walker, Joseph R. Masiero, Erin K. Blauvelt, Robert S. McMillan, Yan R. Fernández, Karen J. Meech, Carey M. Lisse, Roc M. Cutri, John W. Dailey, David J.

Tholen, Timm Riesen, Laurie Urban, Alain Khayat, George Pearman, James V. Scotti, Emily Kramer,

De'Andre Cherry, Thomas Gautier, Stephanie Gomillion, Jessica Watkins, and Edward L. Wright 


\title{
WISE/NEOWISE OBSERVATIONS OF ACTIVE BODIES IN THE MAIN BELT
}

\author{
James M. Bauer $^{1,2}$, A. K. Mainzer ${ }^{1}$, Tommy GraV ${ }^{3}$, Russell G. Walker ${ }^{4}$, Joseph R. Masiero ${ }^{1}$, Erin K. Blauvelt ${ }^{1}$, \\ Robert S. McMillan ${ }^{5}$, Yan R. Fernández ${ }^{6}$, Karen J. Meech $^{7,8}$, CArey M. Lisse ${ }^{9}$, Roc M. Cutri ${ }^{2}$, John W. Dailey ${ }^{2}$, \\ David J. Tholen ${ }^{7}$, Timm Riesen ${ }^{7}, 8$, Laurie Urban $^{7}$, Alain Khayat $^{7}$, GeOrge Pearman $^{2}$, James V. Scotti $^{5}$, Emily Kramer ${ }^{6}$, \\ De’Andre Cherri ${ }^{1}$, Thomas Gautier ${ }^{1}$, Stephanie Gomillion $^{1}{ }^{1}$ Jessica Watkins $^{1}$, Edward L. Wright ${ }^{10}$ \\ AND THE WISE TEAM \\ ${ }^{1}$ Jet Propulsion Laboratory, California Institute of Technology, 4800 Oak Grove Drive, MS 183-401, Pasadena, CA 91109, USA; bauer@ scn.jpl.nasa.gov \\ ${ }^{2}$ Infrared Processing and Analysis Center, California Institute of Technology, Pasadena, CA 91125, USA \\ ${ }^{3}$ Planetary Science Institute, 1700 East Fort Lowell, Suite 106, Tucson, AZ 85719-2395, USA \\ ${ }^{4}$ Monterey Institute for Research in Astronomy, 200 Eighth Street, Marina, CA 93933, USA \\ ${ }^{5}$ Lunar and Planetary Laboratory, University of Arizona, 1629 East University Boulevard, Kuiper Space Science Building 92, Tucson, AZ 85721-0092, USA \\ ${ }^{6}$ Department of Physics, University of Central Florida, 4000 Central Florida Boulevard, P.S. Building, Orlando, FL 32816-2385, USA \\ ${ }^{7}$ Institute for Astronomy, University of Hawaii, 2680 Woodlawn Drive, Manoa, HI 96822, USA \\ ${ }^{8}$ NASA Astrobiology Institute, University of Hawaii, Manoa, HI 96822, USA \\ ${ }^{9}$ Applied Physics Laboratory, Johns Hopkins University, 11100 Johns Hopkins Road, Laurel, MD 20723-6099, USA \\ ${ }^{10}$ Department of Physics and Astronomy, University of California, PO Box 91547, Los Angeles, CA 90095-1547, USA \\ Received 2011 September 13; accepted 2011 December 13; published 2012 February 13
}

\begin{abstract}
We report results based on mid-infrared photometry of five active main belt objects (AMBOs) detected by the Wide-field Infrared Survey Explorer (WISE) spacecraft. Four of these bodies, P/2010 R2 (La Sagra), 133P/ElstPizarro, (596) Scheila, and 176P/LINEAR, showed no signs of activity at the time of the observations, allowing the WISE detections to place firm constraints on their diameters and albedos. Geometric albedos were in the range of a few percent, and on the order of other measured comet nuclei. P/2010 A2 was observed on 2010 April $2-3$, three months after its peak activity. Photometry of the coma at 12 and $22 \mu \mathrm{m}$ combined with ground-based visible-wavelength measurements provides constraints on the dust particle mass distribution (PMD), $d \log n / d \log$ $m$, yielding power-law slope values of $\alpha=-0.5 \pm 0.1$. This PMD is considerably more shallow than that found for other comets, in particular inbound particle fluence during the Stardust encounter of comet 81P/Wild 2. It is similar to the PMD seen for 9P/Tempel 1 in the immediate aftermath of the Deep Impact experiment. Upper limits for $\mathrm{CO}_{2}$ and $\mathrm{CO}$ production are also provided for each $\mathrm{AMBO}$ and compared with revised production numbers for WISE observations of 103P/Hartley 2.
\end{abstract}

Key words: comets: general - minor planets, asteroids: general

Online-only material: color figures

\section{INTRODUCTION}

Until recently no main belt asteroids were ever seen to exhibit dust ejection or cometary activity. However, in 1996 the discovery of activity from 133P/Elst-Pizarro (Elst et al. 1996), and later in multiple other bodies (e.g., Read et al. 2005; Hsieh \& Jewitt 2006; Hsieh 2007), it became clear that a subset of the bodies within the Main Belt exhibited coma, some at regular intervals in their orbit, with the largest activity occurring near their perihelion (cf. Hsieh et al. 2010). As of late 2011, seven bodies in the main belt have been identified as main belt comets (i.e., asteroids with associated dust tails, henceforth referred to as active main belt objects, or AMBOs). Their activity and optical qualities have been well studied (cf. Hsieh et al. 2010, 2011a), but infrared measurements have been reported for only three bodies; Scheila (Tedesco et al. 2002, 2004), and 176P and 133P (Hsieh et al. 2009). Here, we present data taken in the thermal infrared by the Wide-field Infrared Survey Explorer mission (WISE; Wright et al. 2010) of five of these bodies, four of which appeared as point sources, and we provide measurements of their sizes and corresponding albedos. We also present our analysis of the dust surrounding the AMBO P/2010 A2 (LINEAR). Upper limits of $\mathrm{CO}_{2}$ production are also presented and compared with recomputed values of $\mathrm{CO}_{2}$ production for $103 \mathrm{P} /$ Hartley 2 that supersede previously published rates based on WISE fluxes.
The WISE mission surveyed the sky at four mid-IR wavelengths simultaneously, $3.4 \mu \mathrm{m}$ (W1), $4.6 \mu \mathrm{m}$ (W2), $12 \mu \mathrm{m}$ (W3), and $22 \mu \mathrm{m}$ (W4), with approximately 100 times improved sensitivity over the Infrared Astronomical Satellite (IRAS) mission (Wright et al. 2010). The field of view for each exposure was $47 \times 47$ arcmin. Over $99 \%$ of the sky was covered with multiple exposures, averaging 10 per sky region, but varying in density as a function of ecliptic latitude. On the ecliptic, the minimum number of exposures per sky region was 8. As part of an enhancement to the WISE data processing system called "NEOWISE", the WISE Moving Object Processing Software (WMOPS) was developed to find solar system bodies in the WISE images (Dailey et al. 2010; Mainzer et al. 2011a). WMOPS successfully found a wide array of primitive bodies, including Near-Earth Objects (NEOs), main belt asteroids, comets, Trojans, and Centaurs. By the end of the spacecraft mission, NEOWISE identified more than 157,000 small bodies, including 123 comets (Mainzer et al. 2011a). These infrared observations are useful for determining solid body size and albedo distributions, and thermophysical properties such as thermal inertia, the magnitude of non-gravitational forces, and surface roughness (Mainzer et al. $2011 \mathrm{~b}, 2011 \mathrm{c})$. The subset of these bodies exhibiting cometary activity require special treatment in the interpretation of such observations, owing to the material surrounding the solid nucleus, i.e., the contribution to the IR flux from the gas and dust, and the variable nature of the observed brightness of the 
object attributable to outbursts. These IR imaging data provide unique opportunities to characterize four main components of comets: the dust and gas comae, the nucleus, and the extended dust trail. Here we apply these techniques (Bauer et al. 2011) to the five AMBOs detected by WISE: (596) Scheila (hereafter Scheila), 133P/Elst-Pizarro (133P), 176P/LINEAR (176P), P/2010 R2 (La Sagra) (P/2010 R2), and P/2010 A2 (LINEAR) (P/2010 A2). The remaining two known AMBOs, 238P/Read and P/2008 R1 (Garradd), were not detected in any band.

\section{OBSERVATIONS AND ANALYSIS}

The WISE spacecraft surveyed the sky as its terminatorfollowing geocentric polar orbit progressed about $1^{\circ}$ of ecliptic longitude per day. Regular survey operations commenced on 2010 January 14 (MJD 55210), imaging the sky simultaneously in all four bands until the cryogen was depleted in the secondary tank on 2010 August 5 (MJD 55413). The survey then entered a three-band $(W 1-W 3)$ phase that lasted through 2010 September 30 (MJD 55469). The final phase, the post-cryogenic mission with W1 and W2, lasted from 2010 October 1 through 2011 January 31 (MJD 55592; cf. Cutri et al. 2011). All photometric data of detected objects presented here were obtained during the first phase, except for some last-phase observations of Scheila. Some of the imaging data presented here includes observations of objects in the post-cryogenic mission phase.

During the fully cryogenic portion of the mission, simultaneous exposures in the four WISE bands were taken once every $11 \mathrm{~s}$, with exposure durations of $8.8 \mathrm{~s}$ in $W 3$ and $W 4$, and $7.7 \mathrm{~s}$ in $W 1$ and $W 2$ (Wright et al. 2010). The number of exposures acquired on moving objects varied depending on the location of the object on the sky (especially its ecliptic latitude), the toggle pattern of the survey employed to avoid imaging the Moon, and the relative motion of the object with respect to the progression of the survey (Mainzer et al. 2011a; Cutri et al. 2011). Note that WISE may have observed an object while it was in different patches of the sky, i.e., when several weeks or months had passed since the previous exposure; henceforth, we refer to the series of exposures containing the AMBO in the same region of sky as a "visit."

Table 1 lists the exposures for each of the five detected AMBOs, as well as their mean viewing phase angles, and heliocentric and observer distances during each visit. With two separate visits, (596) Scheila had the greatest coverage depth in $W 1$ and $W 2$, while $\mathrm{P} / 2010 \mathrm{R} 2$ had the greatest depth of $W 3$ and $W 4$ coverage. The last four entries of Table 1 also show the frames that covered the predicted locations of $238 \mathrm{P}$ and $\mathrm{P} / 2008$ R1 (Garradd), which were not detected in either the individual or stacked frames, but from which we derive upper limits to their fluxes (see Table 2).

The WISE image data were processed using the scan/frame pipeline that applied instrumental, photometric, and astrometric calibrations (Cutri et al. 2011). Each of our five objects was observed more than the average 10 times owing to the object's (prograde) motion being in the direction of the scan progression of the spacecraft. WISE covered all ecliptic latitudes each day in two narrow longitude bands at $95^{\circ} \pm 2^{\circ}$ ahead of the Sun and $90^{\circ} \pm 2^{\circ}$ behind the Sun, and used the spacecraft orbital motion around the Sun to scan this band across all ecliptic longitudes over six months. P/2010 A2 had the maximum skyplane motion of the five AMBOs with a rate of 42-61 arcsec $\mathrm{hr}^{-1}$ ("moving" mostly via the reflex motion due to parallax and the spacecraft's orbital motion). At most, the apparent motion created a blurring of $\sim 0.15$ arcsec, an insignificant fac- tor in the imaging, as the blur was far smaller than the pixel scale in the shortest wavelengths $\left(2.75 \operatorname{arcsec}_{\text {pixel }}^{-1}\right.$ in $W 1$, $W 2$, and $W 3 ; 5.5 \operatorname{arcsec}$ pixel $^{-1}$ in $2 \times 2$ binned $W 4$ images; Wright et al. 2010), and the point-spread function (PSF) FWHM (6.5 arcsec in the three shortest wavelength bands). Scheila and $\mathrm{P} / 2010$ A2 were detected in individual exposures, with sufficient signal to be detected with NEOWISE moving object pipeline software (WMOPS; Mainzer et al. 2011a). The images of P/2010 A2 were stacked in order to increase the signal-tonoise of the dust tail; the images for P/2010 R2, 176P, and 133P were stacked to increase any signal from these bodies that were present, and the detections of the objects were identified only in the stacked images. The images for the AMBOs were shifted to match the sky-motion rates of each object as predicted by JPL's Horizon's ephemeris service (http://ssd.jpl.nasa.gov), and co-added using the "A WISE Astronomical Image Co-adder" (AWAIC) algorithm as described in Masci \& Fowler (2009). All images were stacked in this manner for each corresponding visit to an object to conduct the photometric and morphological analyses.

For Scheila, 176P, 133P, and P/2010 R2, the image profiles including those of the stacked images were consistent with PSFs, while the profile of $\mathrm{P} / 2010$ A2 was not consistent with a PSF, owing to the presence of coma. Special consideration was given to P/2010 R2 and to the December visit of Scheila (detected in the $W 1$ and $W 2$ bands only), owing to the fact that the WISE observations were relatively close in time to reported activity, or predicted activity (cf. Moreno et al. 2011). No coma signal was apparent in the images (see Figure 1), nor was any significant coma signal found for $\mathrm{P} / 2010 \mathrm{R} 2$ or Scheila when surface brightness profile (SBP) analysis techniques were applied (cf. Bauer et al. 2008). The SBPs shown in Figure 2 were generated from the $W 3$ stacked image for P/2010 R2 and from the W2 stacked image for Scheila during the WISE spacecraft's second visit to the AMBO, i.e., in the post-cryo mission data. The PSFs and AMBO SBPs were sampled out to 30 arcsec, well into the region where each object's SBP wings reach into the local background. Analysis also showed that fluxes for Scheila were consistent with the flux values derived from the 2010 February visit data when re-scaled for distance and phase angle (IAU phase parameter $G=0.08$; Bowell et al. 1989), suggesting that there was no surrounding dust or out-gassing.

Aperture photometry was performed on the stacked images of $176 \mathrm{P}, 133 \mathrm{P}$, and $\mathrm{P} / 2010 \mathrm{R} 2$ for aperture radius values of 11 and 22 arcsec, the aperture sizes necessary to obtain the full signal from $W 3$ and $W 4$, the poorest resolution WISE bands, while pipeline-extracted magnitudes were used for the thermal fits of Scheila. The counts were converted to fluxes using the band-appropriate magnitude zeropoints and 0th magnitude flux values provided in Wright et al. (2010), and an iterative fitting to a black-body curve was conducted on the two long-wavelength bands to determine the appropriate color correction as listed in the same. The extracted magnitudes were then converted to fluxes (Wright et al. 2010; Mainzer et al. 2011b) and are listed in Table 2. Proper aperture corrections are required for accurate photometry (Cutri et al. 2011), in addition to the color corrections mentioned above. With these corrections, the derived magnitudes are equivalent to the profile-derived magnitudes providing there are no artifacts, saturation, or confusion with other sources in the images of the objects. Note that the profile magnitudes for Scheila allowed for a more accurate photometric magnitude in $W 3$, since the core of the image was saturated for this object (cf. Mainzer et al. 
Table 1

Mid-IR Observations of Known Active Main Belt Asteroids

\begin{tabular}{|c|c|c|c|c|c|c|c|}
\hline Object $^{\mathrm{a}}$ & $N^{\mathrm{a}}$ & $\begin{array}{c}R^{\mathrm{a}} \\
(\mathrm{AU})\end{array}$ & $\begin{array}{c}\Delta^{\mathrm{a}} \\
(\mathrm{AU})\end{array}$ & $\begin{array}{l}\alpha^{\mathrm{a}} \\
\left({ }^{\circ}\right)\end{array}$ & Coma? $^{a}$ & Stacked? ${ }^{a}$ & Comments $^{\mathrm{a}}$ \\
\hline (596) Scheila & 10 & 3.93 & 3.14 & 16.8 & No & No & $\ldots$ \\
\hline \multicolumn{8}{|c|}{$\begin{array}{l}\text { MJDa Start Times: 55242.35929406, 55242.49159826, 55242.62390243, 55242.69011816, 55242.82242237, } \\
55242.95472657,55242.95485389,55243.08715810,55243.21946226,55243.35176641\end{array}$} \\
\hline $133 \mathrm{P}$ & 13 & 3.67 & 3.42 & 15.6 & No & Yes & $\ldots$ \\
\hline \multicolumn{8}{|c|}{$\begin{array}{l}\text { MJD Start Times: 55271.99996748, 55272.13239883, 55272.26470277, 55272.39700681, 55272.46309514, } \\
\text { 55272.46322245, 55272.52931076, 55272.59552641, 55272.66161475, 55272.72783039, 55272.79391878, } \\
55272.86013443,55272.99243847,55273.12474251\end{array}$} \\
\hline $\mathrm{P} / 2010 \mathrm{~A} 2$ & 16 & 2.07 & 1.73 & 28.8 & Yes & Yes & $\ldots$ \\
\hline
\end{tabular}

MJD Start Times: 55288.27578843, 55288.40809252, 55288.54039657, 55288.67270062, 55288.80500469, 55288.87122037, 55288.93730874, 55289.00352447, 55289.06961284, 55289.13582852, 55289.20191689, $55289.26813252,55289.40043657,55289.53274063,55289.66504468,55289.79734873$

\begin{tabular}{llllllllll}
\hline $176 \mathrm{P}$ & 16 & 3.15 & 2.99 & 18.6 & No & Yes & $\ldots$
\end{tabular}

MJD Start Times: 55309.28058017, 55309.41288442, 55309.54518866, 55309.67749290, 55309.67762031, $55309.80979715,55309.80992451,55309.87601297,55309.94210145,55309.94222876,55310.00831722$, $55310.07453300,55310.14062147,55310.27292571,55310.40523000,55310.53753425$

\begin{tabular}{llllllllll}
\hline $\mathrm{P} / 2010 \mathrm{R} 2$ & 19 & 2.62 & 2.40 & 22.8 & Yes & Yes & $\ldots$ \\
\hline
\end{tabular}

MJD Start Times: 55356.64184157, 55356.77414537, 55356.90644912, 55357.03875296, 55357.17092939, $55357.17105676,55357.23714494,55357.30323309,55357.36944865,55357.43553689,55357.50175322$, 55357.56784223, 55357.63405781, 55357.76636159, 55357.89853814, 55357.89866545, 55358.03084197, $55358.03096933,55358.16314579$

\begin{tabular}{llllllll}
\hline (596) Scheila & 14 & 3.15 & 2.98 & 18.3 & No & No & $\ldots$
\end{tabular}

MJD Start Times: 55510.88545743, 55511.01763413, 55511.14993825, 55511.34833072, 55511.41441912, 55511.41454644, 55511.48063478, 55511.54672802, 55511.61281640, 55511.61294377, 55511.67903215, $55511.87742472,55512.00972890,55512.14190566$

\begin{tabular}{lllllllll}
\hline $238 \mathrm{P}$ & 32 & 2.86 & 2.69 & 20.7 & $\ldots$ & $\ldots$ & No detection
\end{tabular}

MJD Start Times: 55320.52708031, 55320.65938453, 55320.79168874, 55320.92399296, 55321.05629718, 55321.12251292, 55321.18860130, 55321.25481710, 55321.32090552, 55321.38712131, 55321.45320973, 55321.51942547, 55321.65172964, 55321.78403387, 55323.57007663, 55323.57020395, 55323.70238080, $55323.70250817,55323.83468502,55323.83481234,55323.96711712,55324.09942130,55324.16550962$, 55324.23172537, 55324.29781380, 55324.36402954, 55324.43011796, 55324.49633370, 55324.56242208, $55324.69472630,55324.82703043,55324.95933464$

\begin{tabular}{lllllllll}
\hline $\mathrm{P} / 2008 \mathrm{R} 1$ & 13 & 3.46 & 3.27 & 16.6 & $\ldots$ & $\ldots$ & No detection
\end{tabular}

MJD Start Times: 55263.26917722, 55263.40148125, 55263.53378520, 55263.66608919, 55263.73230484, 55263.79839317, 55263.79852053, 55263.86460888, 55263.93082453, 55263.99691291, 55264.12921690, $55264.26164819,55264.39395223$

\begin{tabular}{lllllllll}
\hline $238 \mathrm{P}$ & 15 & 2.46 & 2.17 & 23.6 & $\ldots$ & $\ldots$ & No detection \\
\hline
\end{tabular}

MJD Start Times: 55502.32299741, 55502.45530140, 55502.58747807, 55502.71978206, 55502.78587042, $55502.85208605,55502.91817441,55502.98426272,55502.98439003,55503.05047840,55503.11656671$, 55503.18278234, 55503.31495902, 55503.44726300, 55503.57956699

\begin{tabular}{lllllllll}
\hline $\mathrm{P} / 2008$ & $\mathrm{R} 1$ & 14 & 3.66 & 3.53 & 15.7 & $\ldots$ & No detection
\end{tabular}

MJD Start Times: 55518.82066059, 55518.95296481, 55519.08514168, 55519.08526905, 55519.21744591, $55519.28353439,55519.28366170,55519.34975019,55519.41583862,55519.48205441,55519.54814285$, $55519.68044707,55519.81262398,55519.94492822$

Notes. Each object is listed per visit (see the text). $N$ refers to the number of exposures, $R$ is the heliocentric distance of the AMBO in AU, $\Delta$ is the observer distance in AU, $\alpha$ is the phase angle in degrees. The "Coma?" column refers to whether there is apparent coma in the images. "Stacked?" indicates whether the analysis was performed on a stacked (co-added) image of the $N$ exposures, to increase the signal from the AMBO, or whether each exposure was individually analyzed; no detection indicates there was no detection in the stacked or individual images. MJD, i.e., the modified Julian date, is defined as JD-2400000.5. The range of dates for each listed visit is as follows (all dates are 2010): (596) Scheila (1st visit)—Feb 15, 05:26:52 to Feb 16, 08:26:33; 133P-Mar 16, 23:59:57 to Mar 18, 02:59:38; P/2010 A2-Apr 2, 06:37:08 to Apr 3, 19:08:11; 176P-Apr 23, 06:44:02 to Apr 24, 12:54:03; P/2010 R2_Jun 9, 15:24:15 to Jun 11, 03:54:56; (596) Scheila (2nd visit)—Nov 10, 21:15:04 to Nov 12, 03:24:21; 238P (1st visit)—May 04, 12:39:00 to May 8, 23:01:27; P/2008 R1 (1st visit)—Mar 8, 06:27:37 to Mar 9, 09:27:17; 238P (2nd visit)—Nov 2, 07:45:07 to Nov 3, 13:54:35; P/2008 R1 (2nd visit)—Nov 18, 19:41:45 to Nov 19, 22:40:42. 




Figure 1. Three-color composite image of AMBOs from the WISE data. The stacked 4.6, 11.6, and $22.1 \mu \mathrm{m}$ images were mapped to blue, red, and green channels. The AMBOs from left to right are (top row) P/2010 A2 and P/2010 R2, and (bottom row) Scheila, 176P, and 133P. The P/2010 A2 image is 9 arcmin across its bottom edge, while the others are 4.5 arcmin across. The $\mathrm{P} / 2010 \mathrm{~A} 2$ panel shows the sky-projected anti-solar vector as indicated by the green dashed arrow, and the projected anti-velocity vector by the white dotted arrow. Note that while Scheila is saturated in W3, the profile photometry used in our analysis is still viable (Cutri et al. 2011). (A color version of this figure is available in the online journal.)

Table 2

Total Fluxes in mJy

\begin{tabular}{lccccc}
\hline \hline Object & $\begin{array}{c}W 1 \\
(3.5 \mu \mathrm{m})\end{array}$ & $\begin{array}{c}W 2 \\
(4.6 \mu \mathrm{m})\end{array}$ & $\begin{array}{c}W 3 \\
(12 \mu \mathrm{m})\end{array}$ & $\begin{array}{c}W 4 \\
(22 \mu \mathrm{m})\end{array}$ & $\log \left(Q_{\mathrm{CO}_{2}} / Q_{\mathrm{co})}\right.$ \\
\hline Scheila cryo & $8.7 \pm 0.5$ & $17 \pm 1$ & $2700 \pm 300$ & $6800 \pm 800$ & $\ldots$ \\
Scheila post-cryo & $11.0 \pm 0.6$ & $30 \pm 2$ & $\ldots$ & $\ldots$ & $\ldots$ \\
133P & $<0.03$ & $<0.05$ & $1.3 \pm 0.2$ & $4.0 \pm 0.6$ & $<25.6,<26.6$ \\
176P & $<0.02$ & $<0.06$ & $3.2 \pm 0.2$ & $8.0 \pm 0.8$ & $<25.5,<26.5$ \\
P/2010 R2 & $<0.01$ & $<0.03$ & $1.33 \pm 0.07$ & $5.2 \pm 0.5$ & $<25.0,<26.0$ \\
P/2010 A2 & $<0.09$ & $<0.11$ & $57 \pm 9$ & $124 \pm 21$ & $<25.3,<26.3$ \\
238P & $<0.02$ & $<0.06$ & $<0.2$ & $<0.5$ & $<25.4,<26.4$ \\
P/2008 R1 & $<0.04$ & $<0.06$ & $<0.2$ & $<0.6$ & $<25.6,<26.6$ \\
\hline
\end{tabular}

$2011 b)$. Table 2 also lists the $1 \sigma$ upper limits of fluxes in $W 1$ and $W 2$ for the remaining AMBOs with PSF-like profiles that lack detections in these bands. Corresponding $\mathrm{CO}_{2}$ and $\mathrm{CO}$ upper limits are also provided in units of $\log \mathrm{mol} \mathrm{s}^{-1}$, based on analysis outlined in Pittichova et al. (2008) and Bauer et al. (2011). The values listed assume a single source species for the observed upper limit in $W 2$. Note that in the course of the analysis, the $\mathrm{CO}_{2}$ and $\mathrm{CO}$ values were re-computed for $103 \mathrm{P} /$ Hartley 2, and were found to be off by a factor of 17 as reported in Bauer et al. (2011) when using a higher precision code. The corrected column densities for $\mathrm{CO}_{2}$ and $\mathrm{CO}$ are 3.0 $( \pm 2.1) \times 10^{11}$ and $3( \pm 2) \times 10^{10} \mathrm{~m}^{-2}$, respectively. Production values therein should also be corrected as $6.0( \pm 2.0) \times 10^{25}$ and $6.5( \pm 2.2) \times 10^{24} \mathrm{~mol} \mathrm{~s}^{-1}$ for $\mathrm{CO}_{2}$ and $\mathrm{CO}$, respectively. The relative production rates for $\mathrm{CO}_{2}$ as compared to the predicted level of water production, then, are on the order of $20 \%$, rather than the few percent stated in Bauer et al. (2011). The AMBO production upper limits compare with the $103 \mathrm{P} \mathrm{CO}_{2}$ production rate of 25.78 in $\log$ units. These AMBO production upper limits are weak constraints, owing to the greater distance of
Table 3

Object Nucleus Thermal Fits

\begin{tabular}{lcccc}
\hline \hline Object & $\begin{array}{c}\text { Diameter } \\
(\mathrm{km})\end{array}$ & $p_{\mathrm{v}}$ & $p_{\text {ir }}$ & $\eta$ \\
& $118 \pm 6$ & $0.04 \pm 0.004$ & $0.08 \pm 0.03$ & $0.83 \pm 0.03$ \\
Scheila & $3.2 \pm 0.2$ & $0.06 \pm 0.02$ & $0.12 \pm 0.03$ & $0.8 \pm 0.1$ \\
$133 \mathrm{P}$ & $3.5 \pm 0.1$ & $0.07 \pm 0.03$ & $0.15 \pm 0.05$ & $0.8 \pm 0.1$ \\
$176 \mathrm{P}$ & $2.8 \pm 0.3$ & $0.01 \pm 0.01$ & $0.02 \pm 0.02$ & $1.9 \pm 0.3$ \\
$\mathrm{P} / 2010 \mathrm{R} 2$ & $1.6 \pm 0.3$ & $0.03 \pm 0.02$ & $0.05 \pm 0.03$ & Fixed at 0.8 \\
$\mathrm{P} / 2010 \mathrm{R} 2$ & $\leqslant 1.2$ & $\geqslant 0.03$ & $\ldots$ & Fixed at 0.8 \\
$238 \mathrm{P}$ & $\leqslant 1.1$ & $\geqslant 0.04$ & $\ldots$ & Fixed at 0.8 \\
P/2008 R1 & & & &
\end{tabular}

the AMBOs from WISE, relative to 103P, at each object's times of observation. The AMBO flux upper limits are on the order of the expected confusion limits for each band (Cutri et al. 2011), as are the upper limits derived from the stacked images of 238P and $\mathrm{P} / 2008 \mathrm{R} 1$. Using the thermal fit parameters for $176 \mathrm{P}$, we find $3 \sigma$ upper limits of $2.0 \mathrm{~km}$ and $1.8 \mathrm{~km}$ (or $1 \sigma$ limits of 1.2 and $1.1 \mathrm{~km}$ ) for $238 \mathrm{P}$ and $\mathrm{P} / 2008 \mathrm{R} 1$, respectively. We list the $1 \sigma$ upper limits in Table 3, and note that the limit is consistent with 

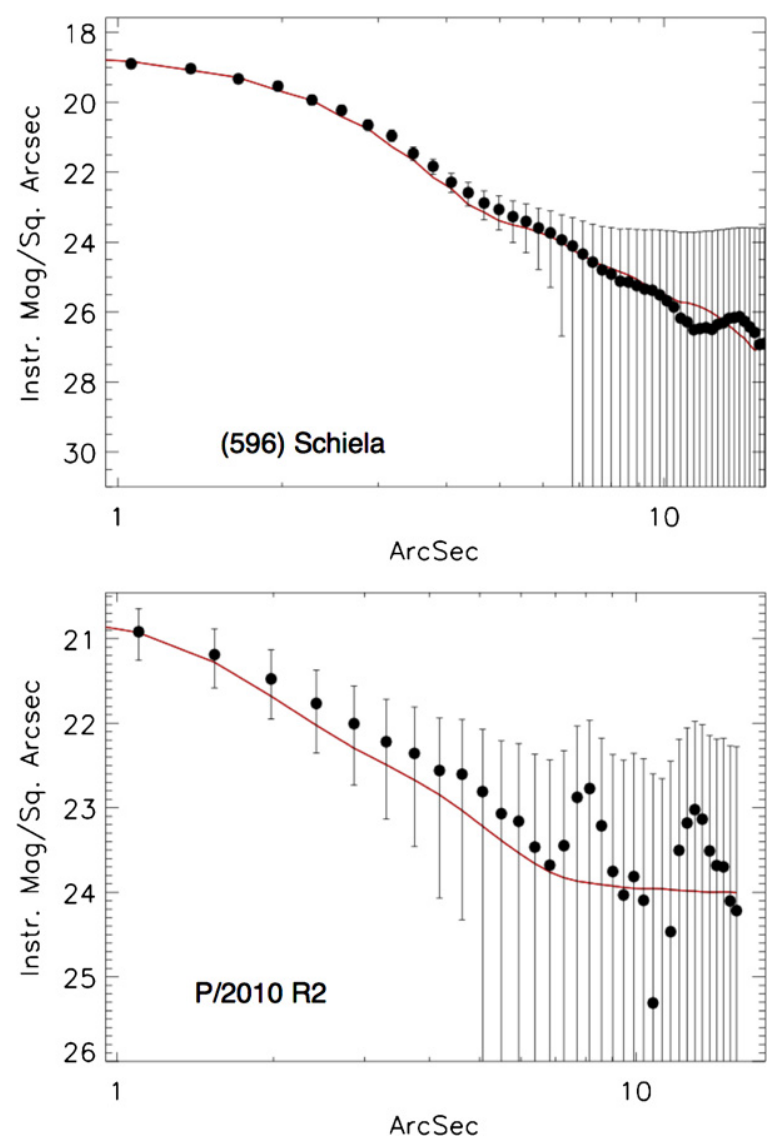

Figure 2. Surface brightness profiles for Scheila in $W 2$ (top panel) and P/2010 $\mathrm{R} 2$ in $W 3$ (bottom panel) sampled out to 24 arcsec. The PSFs were constructed from a nearby bright star in the images for P/2010 R2. For Scheila, which had no nearby stellar counterpart of similar brightness, the comparison PSF was constructed from the array of synthetic PSFs available from the WSDS (Cutri et al. 2011), which oversample the PSF variability across the chip. The PSFs that were co-added to form the comparison PSF were appropriately selected based on the pixel location of Scheila on each image. Error bars shown include the photometric uncertainties of the objects as well as the uncertainty in the background level. Note that owing to the considerably lower signal-to-noiseratio for P/2010 R2, the SPB is more coarsely sampled, i.e., binned over twice the interval than that used for Scheila. Magnitude values are instrumental, based on image counts and default zero points (cf. Wright et al. 2010), uncorrected for color.

(A color version of this figure is available in the online journal.)

the 238P diameter estimate of $0.8 \mathrm{~km}$ by Hsieh et al. (2011b) and that the region of sky containing the AMBO was observed by WISE during the reported time of inactivity.

To constrain the albedo, $H_{\mathrm{V}}$ values were obtained from the literature for 176P (Hsieh et al. 2011a), 133P (Hsieh et al. 2010), and Scheila (cf. Tedesco et al. 2004). 176P and 133P have wellcharacterized rotation periods of 22.2 and $3.47 \mathrm{hr}$ (Hsieh et al. 2009, 2010), respectively. The WISE images sampled $\sim 1.3$ rotations for $176 \mathrm{P}$, and $\sim 7.7$ rotations for $133 \mathrm{P}$. The closest match to the WISE data's sampling cadence and the rotation period is for 133P. WISE orbits once every $\sim 95$ minutes (or two orbits every $3.1753 \pm 0.0003 \mathrm{hr}$ as indicated by the times in Table 1), while the reported rotation period for $133 \mathrm{P}$ is 3.47 (1) hr. Each point is sampled $\sim 8 \%$ of the period off its previous nearest sample point in the rotational phase. For 13 visits (not counting the double-sample where 133P's image fell in the frame overlap region near time MJD 55272.463), this covers $96 \%$ of the range of phase space at roughly $8 \%$ intervals. Assuming there is a small bias in the selection of data points, the reported amplitude is $\sim 0.1 \mathrm{mag}$, which is not far off from the fit uncertainty reported in Table 3 . Note that even with the $<1 \%$ rotation period uncertainty reported in Hsieh et al. (2010), it is not possible to extrapolate the rotational phase from the reported observations of 2007. For each visit of Scheila, the span of observations, $\sim 24$ and 30 hours, was well over a full rotation period of $15 \pm 5 \mathrm{hr}$ (cf. Warner et al. 2009). For matching visual-band observations of P/2010 A2, we reduced Spacewatch data from the $0.9 \mathrm{~m}$ Steward Observatory telescope (cf. Larsen et al. 2007) for the night of 2010 March 15, preceding the WISE observations by about 20 days. Using on-frame sources listed in the USNO-1B catalog (Monet et al. 2003) to conduct relative photometry we found a $V$ magnitude of $18.3 \pm 0.4$. We compared the derived values with those from Jewitt et al. (2011a) and found that the magnitudes matched those taken within a day of our 2010 March 15 observations. Jewitt et al. (2011a) also listed reported magnitudes within hours of the WISE April observations, with total magnitude $H_{\mathrm{v}}=14.4$, and we used this value to constrain our visual-band brightness for $\mathrm{P} / 2010 \mathrm{~A} 2$. The only publicly available values for P/2010 R2 were from the Minor Planet Center (http://www.minorplanetcenter.net), which listed the $H_{\mathrm{v}}$ value as 15.1. However, project NEAT observes the predicted position of the AMBO on 2002 February 9 from the Palomar 48 inch, when the predicted $R$-band brightness was 20.1. The three images were provided by the NEAT archive project (cf. Lawrence et al. 2009) and were stacked matching the predicted sky motion of the AMBO. A signal-to-noise ratio of 4 was achieved down to $m_{\mathrm{R}}=20.5$, and no corresponding source was found near the predicted location. Therefore, we instead used an $H_{\mathrm{V}}$ for P/2010 R2 derived from observations taken at the University of Hawaii 2.2 m telescope on Mauna Kea, HI on 2011 August 1 and 9 (UT) in place of the MPC's listed value. These observations yielded an estimated magnitude of $23.8 \pm$ 0.1 in $R$ band $\left(R=3.0 \mathrm{AU}, \Delta=3.5 \mathrm{AU}, \alpha=16^{\circ}\right)$, corresponding to the $H_{\mathrm{v}} \sim 18.0$, assuming an IAU phase slope parameter of $G=0$, and near-solar colors. This was the best available estimate; those at the MPC were based on observations obtained close to the time of apparent onset of activity. The measured colors of 133P (Hsieh et al. 2010), 176P (Hsieh et al. 2009), and (596) Scheila (pre-outburst; Tedesco 1995, Yang \& Hsieh 2011) are near-solar, within $0.06 \mathrm{mag}$, and $G$ parameters are in the range of -0.1 to 0.1 . Assuming an offset in both values ( $G=0.1$ and $V-R=0.42$ ), the magnitude offset would be $\sim 0.12$, or approximately $12 \%$ in brightness, in both cases, considerably less than the uncertainty in albedo listed in Table 3, and on the order of the photometric uncertainty.

\section{DISCUSSION}

Using an NEATM model (Harris 1998; Mainzer et al. 2011b) thermal fits were conducted on the photometric fluxes of the AMBOs without apparent coma (Figure 3); the fits are summarized in Table 3 . We present here the fit results and uncertainties, while it should be noted that, owing to the uncertainty in the absolute calibration of the WISE thermal bands, there is an additional $\sim 10 \%$ uncertainty in the derived diameter values (Mainzer et al. 2011b, 2011c). Hsieh et al. (2009) report sizes for 133P and 176P based on the Spitzer Space Telescope MIPS $24 \mu \mathrm{m}$ signal alone. The sampling cadence of the MIPS data consisted of three exposures taken over an 8 minute interval for 133P and two exposures spaced $5 \mathrm{hr}$ apart for 176P. As discussed previously, the WISE observations consisted of 13 and 16 visits for $133 \mathrm{P}$ and $176 \mathrm{P}$, respectively, spaced at $1.59 \mathrm{hr}$ intervals and with a more complete sampling of each 

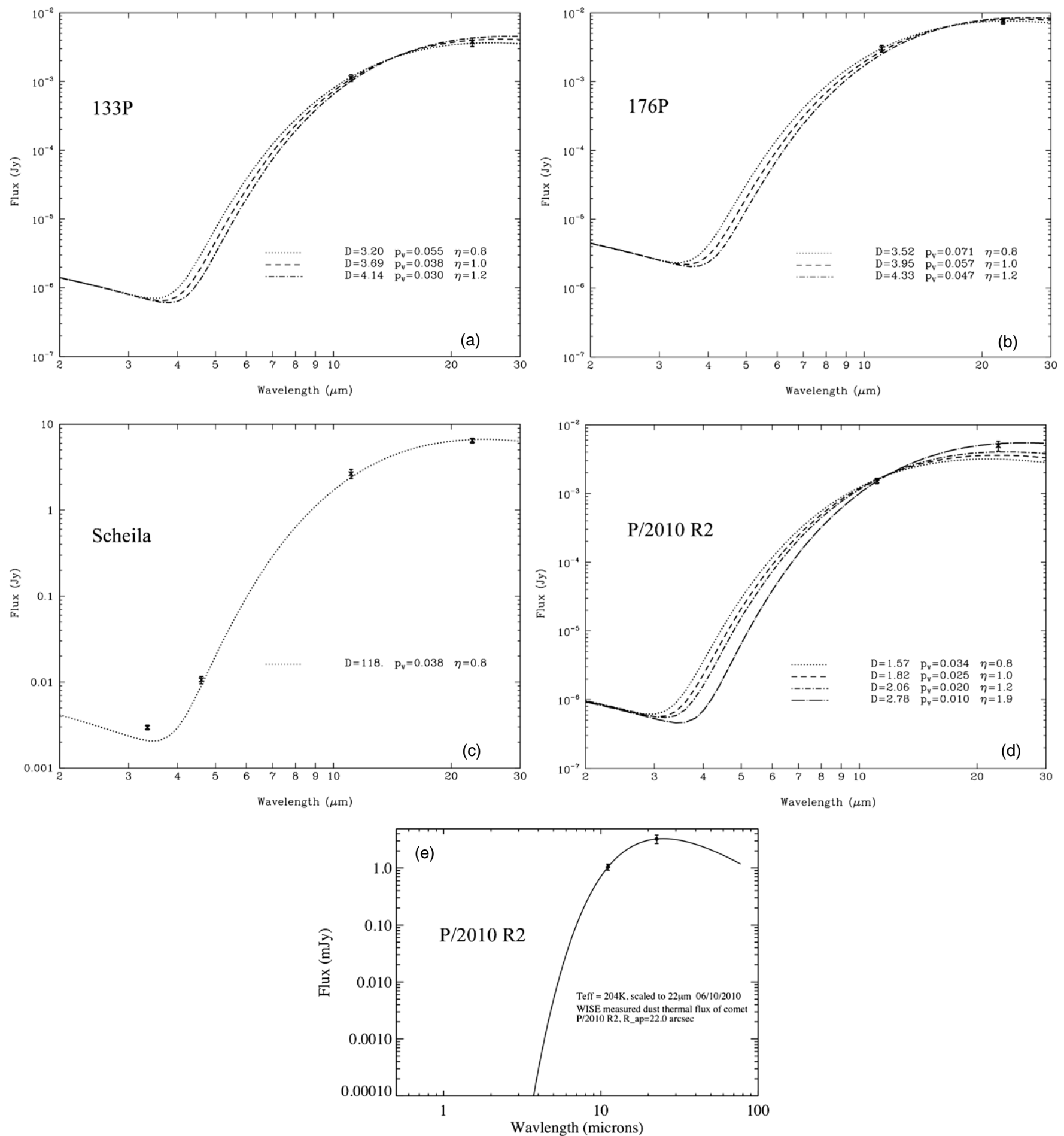

Figure 3. Thermal fits for the AMBOs exhibiting PSF-shaped profiles: 133P (panel A), 176P (panel B), Scheila (panel C), and P/2010 R2 (panels D and E). Panels A-D show fits using the NEATM model appropriate for signal dominated by solid nuclei (Harris 1998; Delbo \& Harris 2002; Mainzer et al. 2011b), with beaming parameter values of $\eta=0.8$ (dotted lines), $\eta=1.0$ (dashed lines), and $\eta=1.2$ (dot-dashed lines). Note that in panel $\mathrm{C}$, the fit to 596 converged freely to $\eta=0.8$ (see Table 3 for the best-fit parameters), and that for panel $\mathrm{D}$ we include the best-fit model of $\eta=1.9$ (dot-long-dashed line); the $\eta$ values were otherwise fixed for the fits shown. The derived diameters $\left(D\right.$ (km units)) and albedos $\left(p_{\mathrm{v}}\right)$ are also shown in the lower right of each of these panels for each model fit. Panel $\mathrm{E}$ shows the black-body fit to P/2010 R2, appropriate for a dust-coma dominated signal, through no apparent extended profile was found in the stacked image (see the text).

body's rotational phase. WISE observed both bodies far from the heliocentric distances about their perihelion where their activity was previously reported (Hsieh et al. 2010, 2011a), and comparable to the distances of the Spitzer Space Telescope observations reported in Hsieh et al. (2009). The WISE data had two thermal channels at 12 and $22 \mu \mathrm{m}$, which allowed for a fit with $\eta$ as a free parameter, and the fits converged to solutions of $\eta$ near 0.8 . Considering these factors, the WISE results of size and albedo compare well with the Hsieh et al. (2009) results for the fixed $\eta \sim 0.8$. Converting the Hsieh et al. (2009) values of $p_{\mathrm{R}}$ to $p_{\mathrm{V}}$, we derive for $133 \mathrm{P}$ an albedo of $p_{\mathrm{V}}=0.04 \pm 0.01$, and for $176 \mathrm{P}, p_{\mathrm{V}}=0.05 \pm 0.01$, which overlap with our values 


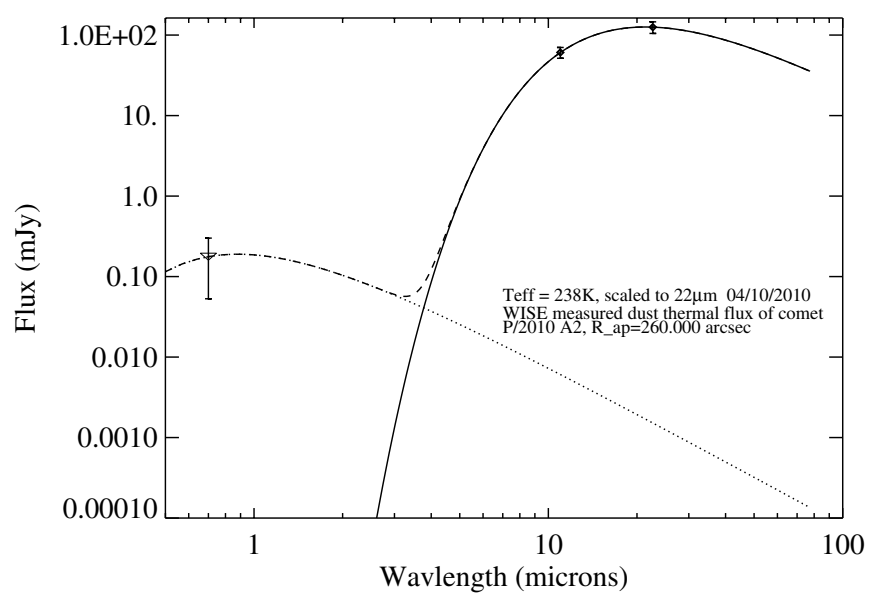

Figure 4. Coma temperature fit to the 4.3 arcmin aperture thermal photometry in the two longest WISE wavelength bands of the P/2010 A2 observations. A reflected-light model with a neutral reflectance (heavy dotted) based on the near-simultaneous photometry from Jewitt et al. (2011a) is shown along with the combined signal (dashed line). The uncertainties to the temperature fit are on the order of $\pm 9 \mathrm{~K}$, and the fitted temperature $(238 \mathrm{~K})$ is in excess of the black body temperature for that distance $(200 \mathrm{~K})$.

in Table 3. Hsieh et al. (2009) report sizes ranging from 3.34 to $3.56 \mathrm{~km}$ for $133 \mathrm{P}$ and $3.44-4.08 \mathrm{~km}$ for $176 \mathrm{P}$ using $\eta=0.8$ calculations, which overlap our derived values and uncertainties, although our sample likely falls closer to the mean, based on the WISE imaging cadence for each object.

We used a default value of 2 for the ratio of $p_{\mathrm{IR}} / p_{\mathrm{V}}$ in the thermal fit results. Note that the fits to this ratio are only loosely constrained by the $W 3$ and $W 4$ signal for $133 \mathrm{P}, 176 \mathrm{P}$, and $\mathrm{P} / 2010 \mathrm{R} 2$, and so the value of $p_{\mathrm{IR}} / p_{\mathrm{V}}$ is close to the default value used. For Scheila, however, $p_{\mathrm{IR}} / p_{\mathrm{V}}$ was strongly constrained by the additional $W 1$ and $W 2$ signal, so that the ratio of 2 is a firmly fitted result, but not unlike what has been found for the WISE data for other redder $(V-R>0.36)$ main belt objects (cf. Mainzer et al. 2011b), as in the case of those in the Scheila D-type spectral class (Yang \& Hsieh 2011).

Physical parameters derived from the WISE data differ between the dust of active coma and solid nucleus surfaces. The general properties of the WISE data have been discussed in detail by Cutri et al. (2011), and the performance of thermal models applied to WISE observations of solid bodies is described in Mainzer et al. (2011b, 2011c). Methods used in the analysis of the coma dust particles of $\mathrm{P} / 2010 \mathrm{~A} 2$ were similar to these introduced in Bauer et al. (2007, 2008, 2011). Analysis of the flux of coma constrains the dust particle size distribution and the quantity of $\mathrm{CO}$ and $\mathrm{CO}_{2}$ emitted by the comet. The IR fluxes for solid nuclei provide constraints on the size of the comet and, when accompanied by shorter (non-thermal) wavelength data, constrain surface albedo values as well.

P/2010 A2 was the only AMBO in our sample to exhibit an apparent dust tail while WISE observed the body. As the coma dominated the signal, no special extraction of the nucleus signal was possible. Thermal fits to the coma of P/2010 A2 were conducted using a Planck function (Figure 4), similar to the analysis conducted on 103P/Hartley 2 (Bauer et al. 2011). The contribution of the nucleus was not removed in this case, as the signal from the nucleus, predicted to be $\sim 120 \mathrm{~m}$ across (Jewitt et al. 2010; Snodgrass et al. 2010), was less than $2 \%$ of the total signal, and WISE was unable to resolve it separately from the dust signal. The best thermal fit to a Planck function is shown in Figure 4 and yields a higher temperature $(238 \mathrm{~K}$ )
Table 4

Particle Mass Distribution

\begin{tabular}{llll}
\hline \hline Quantity & \multicolumn{1}{c}{ R Band } & \multicolumn{1}{c}{$12 \mu \mathrm{m}$} & \multicolumn{1}{c}{$22 \mu \mathrm{m}$} \\
\hline$m_{\mathrm{g}}(\mathrm{kg})$ & $1.8 \times 10^{-16}$ & $8.1 \times 10^{-13}$ & $5.7 \times 10^{-12}$ \\
$n_{\mathrm{g}}$ & $1.3 \times 10^{28}$ & $5.0 \times 10^{15}$ & $1.4 \times 10^{16}$ \\
\hline
\end{tabular}

than the expected black-body temperature (200 K) for $\mathrm{P} / 2010$ A2's solar distance. One possible explanation for this is that the coma is dominated by very small (a few $\mu \mathrm{m}$ ) grains that are highly absorbing in the optical but poorly emitting in the far-IR. Small grains are also likely to evince silicate emission bands (cf. Kolakovola et al. 2004), in which case the W3 excess is $\sim 30 \%$ of the signal, provided the grains are nearly the same temperature as a black body. Another more likely possibility, that the grains are not isothermal emitters, which may be the case for large $(>1 \mathrm{~cm})$ grain sizes, is supported on dynamical grounds from the imagery of Jewitt et al. (2011a), Snodgrass et al. (2010), and Moreno et al. (2010). Since, as in Figure 1, the $\mathrm{P} / 2010 \mathrm{~A} 2$ dust follows the $\mathrm{P} / 2010 \mathrm{~A} 2$ orbit closely, larger $(>100 \mu \mathrm{m})$ grains are likely dominant. While very near its perihelion (perihelion was on 2009 December 4 at a heliocentric distance of 2.0055 AU, outburst discovery heliocentric distance on 2009 December 15 was $2.0061 \mathrm{AU})$, the activity in this AMBO is believed to have been initiated by an impact event (Jewitt et al. 2010), though some uncertainty may remain (Moreno et al. 2010). As with all the AMBOs except for Scheila, no $W 1$ or $W 2$ signal was observed in either the individual or stacked images of $\mathrm{P} / 2010 \mathrm{~A} 2$, therefore no estimates of $\mathrm{CO}$ or $\mathrm{CO}_{2}$ production could be derived, but only upper limits. The near-simultaneous Hubble Space Telescope (HST) photometry (within hours of the WISE observations; Jewitt et al. 2011a) provides constraints on the reflected-light dust signal. Using a method identical to that described in Bauer et al. (2011), scaling by the effective projected area of the dust in the coma, we were able to find the number of dust particles $\left(n_{\text {dust }}\right)$ contributing to the signal in the photometry aperture for each wavelength interval (Table 4). Assuming a new particle size in each band similar to the wavelength scale, and subtracting the contribution from each of the preceding band-centered wavelengths, starting with the longest (W4), we derived a particle mass distribution (PMD; $d \log n / d \log m$, with $m$ being the particle mass with constant density $\rho=1 \mathrm{~g} \mathrm{~cm}^{-3}$ ) as shown in Figure 5. A log-log slope was fit to the result, for a comparison with other comets, and found to be $\alpha=-0.5 \pm 0.1$ (note that the aforementioned $W 3$ excess does not significantly affect this fit), considerably more shallow than most active comets, which more commonly fall within the $\alpha=$ -0.8 to -1.2 range (Lisse et al. 1998, 2004, 2007; Fulle 2004); for comparison, divide the values therein by 3 . The particle size distribution slope parameter values computed therein are scaled with respect to size, rather than mass, resulting in a factor of three steeper than ours). Our slope value is instead closer to the slope seen in the immediate post-impact of the Deep Impact experiment on 9P/Tempel 1 (cf. Bauer et al. 2007), consistent with a PMD caused by an impact-driven event. However, an alternative explanation is more likely; a large number of the finer particles may have been driven away from the coma by the solar radiation pressure over the course of the 15 weeks since the first observed activity, or 6-11 months since the projected onset of activity (Jewitt et al. 2010; Snodgrass et al. 2010; Moreno et al. 2010). In that case, no new small particles would have been produced to replace those swept away, implying again a 


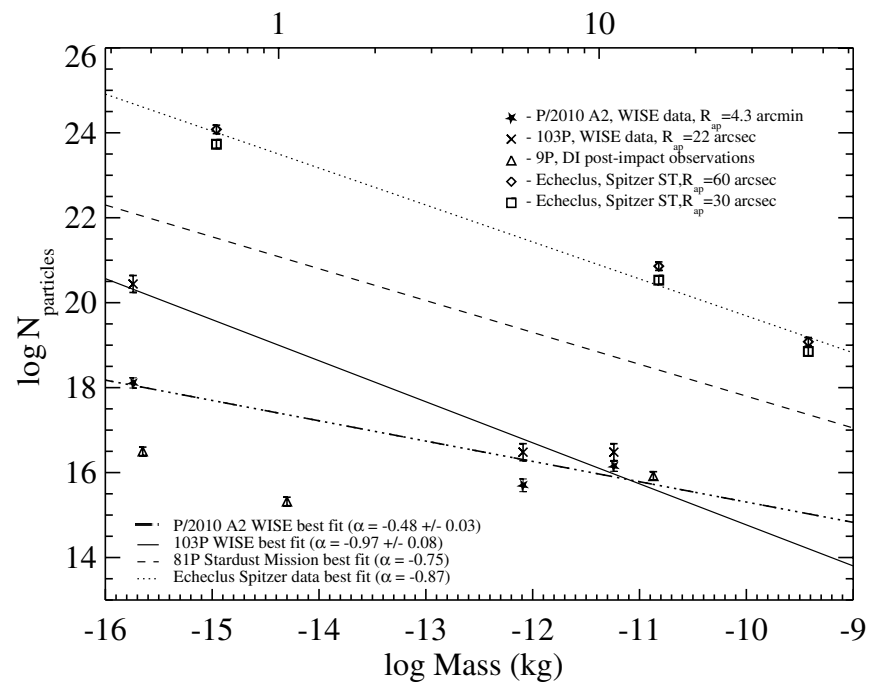

Figure 5. Particle mass distribution (PMD) of P/2010 A2. Log number is shown vertically, while log mass is shown on the bottom scale and the corresponding grain radius size, in microns, is shown on the scale above. The $\mathrm{P} / 2010$ A2 data derived number of particles in the 4.3 arcmin aperture radius (stars), encompassing the complete signal from the dust tail, are shown. For comparison, 103P/Hartley 2 (pentagons; Bauer et al. 2011), Deep Impact particle densities (triangles; Schleicher et al. 2006; Bauer et al. 2007; Lisse et al. 2007), and Echeclus particle numbers (diamonds and squares; Bauer et al. 2008) are also shown. Stardust PMD slope $(\alpha=-0.75$, in $\log N / \log \mathrm{kg}$ units, where $N$ is the estimated total number of dust grains in the aperture; Green et al. 2004) is shown as the dashed line, rescaled from dust fluence values to an aperture encompassing a similar $\rho$ size. Echeclus' PMD best-fit $(\alpha=-0.87)$ is shown as a dotted line, and the solid line is the best fit to 103P PMD data $(\alpha=-0.97)$. The dot-dashed line represents the best fit to the P/2010 A2 data of $\alpha=-0.5$.

sudden outburst limited in duration, and not extending into the epoch of the WISE observations.

Objects 133P, 176P, P/2010 R2, and Scheila all demonstrate image morphologies that matched the observational stellar PSFs. 176P and 133P were not close to their perihelia, i.e., when the bodies were observed to have been the most active in the past (cf. Hsieh et al. 2010, 2011a). However, P/2010 R2 was approaching its perihelion distance of $2.62 \mathrm{AU}$ and was observed to be active 65 days after WISE observed the object. It is still possible that $\mathrm{P} / 2010 \mathrm{R} 2$ was undergoing low-level activity at the time of the WISE observations in late June. The fact that the bestfit beaming parameter for $\mathrm{P} / 2010 \mathrm{R} 2$ is 1.9 is suggestive, though other fits with lower $\eta$ values are still feasible (see Figure 3(D)). Fits with $\eta$ in the range of $0.8-1.2$, although poorer, fall within the $95 \%$ confidence level and produce geometric albedo values in the range of $2 \%-3.4 \%$, closer to the $4 \%-6 \%$ values of other AMBOs, rather than the lower $1 \%$ value for the best fit. We listed the P/2010 R2 fit for the fixed- $\eta$ value of 0.8 in Table 3 in addition to the free- $\eta$ fit for comparison with the other three AMBO fits, all of which yield $\eta$ values near 0.8 . For comparison, Fernandez et al. (2008) find the mean $\eta$ value in their Jupiter family comet sample to be near 1.0, with a standard deviation of 0.1 , significantly less than the high- $\eta$ best fit for P/2010 R2. The best-fit high $\eta$ value implies that the temperature may be cooler than expected, and it may be cool enough to be explained by the presence of isothermal dust grains. Alternatively, the fit to a Planck function shown in Figure 3(E) is elevated by $27 \mathrm{~K}$ from the black-body temperature, which may be caused by the same phenomena (abundant small or large grains or a pronounced silicate emission feature) discussed for $\mathrm{P} / 2010$ A2, or alternatively caused by a signal dominated more by the nucleus. If so, the nucleus size derived from the thermal fits would serve more as an upper limit, since activity would likely enhance the IR flux. The fact that the object shows no sign of activity in WISE data from SBP analysis could be at least partially due to the large pixel scales of the WISE data. The use here of an optical magnitude inferred from actual inactive data could have resulted in an underestimate the true optical magnitude at the time of the WISE observations, leading to an underestimated albedo.

The limits of activity for Scheila are more firmly constrained in that the object size derived from the thermal flux, and the corresponding albedo, match those found in the literature (cf. Tedesco et al. 2004). Larson (2010) mentions a possibly star-like appearance as late as November 11, we provide true photometric and surface brightness constraints on the activity beyond this date. Furthermore, the $\mathrm{W} 1$ data are consistent with a lack of activity when they are corrected for distance and phase angle. For each visit in February and November, the $W 1$ signal is dominated by reflected light for distances $\sim 3 \mathrm{AU}$, and are equal with each other to within $<1 \%$ when corrected for heliocentric and observer distances listed in Table 1, and the observational phase angle change from $18.3^{\circ}$ to $16.8^{\circ}$, using an IAU slope parameter of $G=0.08$. Hence, a clear and closer constraint is placed on the time of Scheila's outburst, within 21 days of the earliest reported activity when the AMBO was $\sim 1.3$ mag in excess brightness on 2010 December 3 (Jewitt et al. 2011b; Bodewits et al. 2011).

The average AMBO albedo derived from WISE observations taken from these data, is 0.06 , and the standard deviation in the sample is 0.02. This mean is consistent with other measurements of AMBO albedos (Hsieh et al. 2009) and is more or less consistent with measured comet reflectances which are $\sim 0.04$ (cf. Lamy et al. 2004). Objects 133P and 176P have been noted as having dynamical and physical similarities with the Themis family asteroids (cf. Hsieh et al. 2009), including similar albedo values. Our own albedo values for $133 \mathrm{P}(0.06 \pm 0.02)$ and $176 \mathrm{P}$ $(0.07 \pm 0.03)$ affirm this comparison, and match the values WISE has measured for the subset of Themis members in Masiero et al. (2011), which has a mean of $0.07 \pm 0.02$.

\section{CONCLUSIONS}

WISE has managed to sample the majority of the known AMBOs in the thermal and mid-IR. One AMBO, P/2010 A2, was dominated by its dust-coma signal, while the others were not likely active at the time of their observations. From the observed fluxes we conclude the following.

1. The thermal fits for $\mathrm{P} / 2010 \mathrm{~A} 2$ yield higher temperature Planck functions than the black body temperature at the observed solar distance by $20 \%(38 \mathrm{~K})$, which can most readily be explained by large, non-isothermal grain dust. The slope of the PMD, in units of $d \log n / d \log m$ is $-0.5 \pm$ 0.1 . The PMD of $\mathrm{P} / 2010 \mathrm{~A} 2$, when fit with a power law, is similar to that seen in an impulsive outburst, but most likely indicates that the activity was over a finite window of time several months in the past.

2. The onset time of activity for Scheila is further constrained by our data to be within 21 days of the first observation of activity. The derived surface reflectance and diameter are consistent with literature values $\left(p_{\mathrm{V}}=0.04 \pm 0.008, D=\right.$ $115 \pm 6$; cf. Tedesco et al. 2004). While the albedo and thermal inertia are entirely in line with canonical cometary values, the derived diameter is one of the largest values measured for a comet. Scheila is apparently larger than 
many of the outer planet's smaller moons and the median diameter of main belt asteroids (cf. Bottke et al. 2005).

3. AMBO nuclei albedos are consistent with measured comet albedos, i.e., are on the order of a few percent.

This publication makes use of data products from the Widefield Infrared Survey Explorer, which is a joint project of the University of California, Los Angeles, and the Jet Propulsion Laboratory/California Institute of Technology, funded by the National Aeronautics and Space Administration. This publication also makes use of data products from NEOWISE, which is a project of the Jet Propulsion Laboratory/California Institute of Technology, funded by the Planetary Science Division of the National Aeronautics and Space Administration. NEAT archive data were provided through NASA's Planetary Mission Data Analysis Program. Observing time was allocated at Steward Observatory's 0.9 m (Spacewatch) telescope on Kitt Peak. J. Bauer also thanks Drs. Hsieh and Jewitt for their valuable discussions regarding AMBOs. This material is based in part upon work supported by the NASA through the NASA Astrobiology Institute under Cooperative Agreement No. NNA09DA77A issued through the Office of Space Science.

\section{REFERENCES}

Bauer, J. M., Choi, Y.-J., Weissman, P. R., et al. 2008, PASP, 120, 393 Bauer, J. M., Walker, R., Mainer, A., et al. 2011, ApJ, 738, 171

Bauer, J. M., Weissman, P. R., Choi, Y-J., et al. 2007, Icarus, 187, 296

Bodewits, D., Kelley, M. S., Li, J.-Y., et al. 2011, ApJ, 733, L3

Bottke, W. F., Jr., Durda, D., Nesvorny, D., et al. 2005, Icarus, 175, 111

Bowell, E., Hapke, B., Domingue, D., et al. 1989, in Asteroids II, Proc. Conf., Application of Photometric Models to Asteroids, Tucson, AZ, 1988 March 8-11, ed. R. P. Binzel, T. Gehrels, \& M. Shapley Matthews (Tucson, AZ: Univ. Arizona Press), 524

Cutri, R. M., Wright, E. L., Conrow, T., et al. 2011, Explanatory Supplement to the WISE Preliminary Data Release Products, http://wise2. ipac.caltech.edu/docs/release/prelim/expsup/

Dailey, J., Bauer, J., Grav, T., et al. 2010, BAAS, 41, 409.04

Delbo, M., \& Harris, A. W. 2002, Meteor. Planet. Sci., 37, 1929

Elst, E. W., Pizarro, O., Pollas, C., et al. 1996, IAU Circ., 6496, 1

Fernandez, Y. R., Kelley, M. S., Lamy, P. L., et al. 2008, in Lunar and Planetary Conf. 1405 (Houston, TX: LPI), 8307

Fulle, M. 2004, in Comets II, Motion of Cometary Dust, ed. M. C. Festou, H. U. Keller, \& H. A. Weaver (Tucson, AZ: Univ. Arizona Press), 565

Green, S. F., McDonnell, J. A. M., McBride, N., et al. 2004, J. Geophys. Res., 109, E12S04

Harris, A. W. 1998, Icarus, 131, 291
Hsieh, H. H. 2007, PhD thesis, Institute for Astronomy, Univ. Hawaii, Manoa Hsieh, H. H., Ishiguro, M., Lacerda, P., \& Jewitt, D. 2011a, AJ, 142, 29

Hsieh, H. H., \& Jewitt, D. C. 2006, Science, 312, 561

Hsieh, H. H., Jewitt, D., \& Fernández, Y. R. 2009, ApJ, 694, L111

Hsieh, H. H., Jewitt, D., Lacerda, P., et al. 2010, MNRAS, 403, 363

Hsieh, H. H., Meech, K. J., \& Pitichova, J. 2011b, ApJ, 736, L18

Jewitt, D., Stuart, J. S., \& Li, J. 2011a, AJ, 142, 28

Jewitt, D., Weaver, H., Agarwal, J., Mutchler, M., \& Drahus, M. 2010, Nature, 467,817

Jewitt, D., Weaver, H., Mutchler, M., Larsen, S., \& Agarwal, J. 2011b, ApJ, 733, L4

Kolakovola, L., Hanner, M. S., Levasseur-Regourd, A.-C., \& Gustafson, B. A. S. 2004, in Comets II, Physical Properties of Cometary Dust from Light Scattering and Thermal Emission, ed. M. C. Festou, H. U. Keller, \& H. A. Weaver (Tucson, AZ: Univ. Arizona Press), 577

Lamy, P. L., Toth, I., Fernandez, Y. R., \& Weaver, H. A. 2004, in Comets II, ed. M. C. Festou, H. U. Keller, \& H. A. Weaver (Tucson, AZ: Univ. Arizona Press), 223

Larsen, J. A., Roe, E. S., Albert, C. E., et al. 2007, AJ, 133, 1247

Larson, S. M. 2010, IAU Circ., 9188

Lawrence, K. J., Bauer, J. M., Meech, K. J., et al. 2009, American Astronomical Society, DPS Meeting 41, 46.03

Lisse, C. M., A’Hearn, M. F., Hauser, M. G., et al. 1998, ApJ, 496, 971

Lisse, C. M., Fernández, Y. R., A’Hearn, M. F., et al. 2004, Icarus, 171, 444

Lisse, C. M., Kraemer, K. E., Nuth, J. A., Li, A., \& Joswiak, D. 2007, Icarus, 187,69

Mainzer, A., Bauer, J., Grav, T., et al. 2011a, ApJ, 731, 53

Mainzer, A., Grav, T., Masiero, J., et al. 2011b, ApJ, 736, 100

Mainzer, A., Grav, T., Masiero, J., et al. 2011c, ApJ, 737, L9

Masci, F. J., \& Fowler, J. W. 2009, in ASP Conf. Ser. 411, AWAIC: A WISE Astronomical Image Co-adder, Astronomical Data Analysis Software and Systems XVIII, ed. D. A. Bohlender, D. Durand, \& P. Dowler (San Francisco, CA: ASP), 67

Masiero, J. R., Mainzer, A. K., Grav, T., et al. 2011, ApJ, 741, 68

Monet, D. G., Levine, S. E., Canzian, B., et al. 2003, AJ, 125, 984

Moreno, F., Lara, L. M., Licandro, J., et al. 2011, ApJ, 738, L16

Moreno, F., Locandro, J., Tozzi, G.-P., et al. 2010, ApJ, 718, L132

Pittichova, J., Woodward, C. E., Kelley, M. S., \& Reach, W. T. 2008, AJ, 136, 1127

Read, M. T., Bressi, T. H., Gehrels, T., Scotti, J. V., \& Christensen, E. J. 2005, IAU Circ., 8624, 1

Schleicher, D. G., Barnes, K. L., \& Baugh, N. F. 2006, AJ, 131, 1130

Snodgrass, C., Tuniaba, C., Vincent, J.-B., et al. 2010, Nature, 467, 814

Tedesco, E. F. 1995, UBV Mean Asteroid Colors, EAR-A-5-DDR-UBVMEAN-VALUES-V1.2, NASA Planetary Data System http://sbn.psi.edu/ pds/resource/imps.html

Tedesco, E. F., Noah, P. V., Noah, M., \& Price, S. D. 2002, AJ, 123, 1056

Tedesco, E. F., Noah, P. V., Noah, M., \& Price, S. D. 2004, IRAS Minor Planet Survey V6.0, NASA Planetary Data System http://sbn.psi.edu/pds/ resource/ubvmean.html

Warner, B. D., Harris, A. W., \& Pravec, P. 2009, Icarus, 202, 134

Wright, E. L., Eisenhardt, P. R. M., Mainzer, A. K., et al. 2010, AJ, 140, 1868

Yang, B., \& Hsieh, H. 2011, ApJ, 737, L39 